\title{
On notch and crack size effects in fatigue, Paris' law and implications for Wöhler curves
}

\author{
M. Ciavarella \\ Politecnico di Bari, Department of Mechanics, Mathematics and Management, Viale Japigia 182, 70126 Bari, Italy \\ mciava@poliba.it
}

\author{
A. Papangelo \\ Politecnico di Bari, Department of Mechanics, Mathematics and Management, Viale Japigia 182, 70126 Bari, Italy \\ Hamburg University of Technology, Department of Mechanical Engineering, Am Schwarzenberg-Campus 1, 21073 Hamburg, \\ Germany \\ antonio.papangelo@poliba.it
}

\begin{abstract}
As often done in design practice, the Wöhler curve of a specimen, in the absence of more direct information, can be crudely retrieved by interpolating with a power-law curve between static strength at a given conventional low number of cycles $\mathrm{N}_{0}$ (of the order of $10-10^{3}$ ), and the fatigue limit at a "infinite life", also conventional, typically $\mathrm{N}_{\infty}=2 \cdot 10^{6}$ or $\mathrm{N}_{\infty}=10^{7}$ cycles. These assumptions introduce some uncertainty, but otherwise both the static regime and the infinite life are relatively well known. Specifically, by elaborating on recent unified treatments of notch and crack effects on infinite life, and using similar concepts to the static failure cases, an interpolation procedure is suggested for the finite life region. Considering two ratios, i.e. toughness to fatigue threshold $F_{K}=K_{I c} / \Delta K_{t h}$, and static strength to endurance limit, $F_{R}=\sigma_{R} / \Delta \sigma_{0}$, qualitative trends are obtained for the finite life region. Paris' and Wöhler's coefficients fundamentally depend on these two ratios, which can be also defined "sensitivities" of materials to fatigue when cracked and uncracked, respectively: higher sensitivity means stringent need for design for fatigue. A generalized Wöbler coefficient, $k$, is found as a function of the intrinsic Wöhler coefficient $k$ of the material and the size of the crack or notch. We find that for a notched structure, $k<k^{\prime}<m$, as a function of size of the notch: in particular, $k^{\prime}=k$ holds for small notches, then $k^{\prime}$ decreases up to a limiting value (which depends upon $K_{t}$ for mildly notched structures, or on $F_{K}$ and $F_{R}$ only for severe notch or crack). A perhaps surprising return to the original slope $k$ is found for very large blunt notches. Finally, Paris' law should hold for a distinctly cracked structure, i.e. having a long-crack; indeed, Paris' coefficient $m$ is coincident with the limiting value of $k^{\prime}$ lim. The scope of this note is only qualitative and aims at a discussion over unified treatments in fatigue.
\end{abstract}

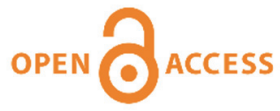

Citation: Ciavarella, M., Papangelo., A., On notch and crack size effects in fatigue, Paris' law and implications for Wöhler curves, 44 (2018) 49-63.

Received: 24.01 .2018

Accepted: 30.01 .2018

Published: 01.04.2018

Copyright: (C) 2018 This is an open access article under the terms of the CC-BY 4.0, which permits unrestricted use, distribution, and reproduction in any medium, provided the original author and source are credited. 
KEYwORDS. Fatigue; Wöhler curve; Notch Sensitivity; Paris' law; Ashby maps.

\section{INTRODUCTION}

I $\mathrm{t}$ is well known that initiation and propagation of cracks are well distinct phenomena, and depend strongly on the material, geometry and load levels (for a review see for example Fleck et al [1]). For nominally plain specimen, at low load levels, where we expect fatigue failure at high cycle numbers (HCF, High Cycle Fatigue) practically the whole life is expended in enucleating the crack, rather than propagating: indeed the latter phase only takes the final few cycles. At high load levels (those giving low number of cycles, LCF), cyclic plastic deformation takes place rapidly leading to failure. These various processes result in the well know empirical Wöhler curve (or, more in detail, in the Basquin-Coffin-Manson's law). Vice versa, for cracked specimen, fatigue life (often denominated "residual") is all given by propagation, generally by Paris's law. The case of notched specimen is somewhere intermediate, and neither Basquin-Coffin-Manson's law nor Paris's laws (nor indeed any other law) apply directly to find the fatigue life. Various alternatives are possible: trying to follow the cyclic plastic deformations at the notch tip (perhaps using Neuber's rule) to link initiation to use Basquin-Coffin-Manson's law at some critical point, and/or integrating Paris' law for a crack once initiated at the crack tip itself. The two processes are not straightforward and certainly at not of the same order of sophistication as the direct use of the Wöhler curve or Paris law as it is possible with plain specimen or cracked ones. Also, some inconsistencies may arise in the procedure, as the use of Paris law requires complications for taking into account of short crack behaviour, crack shielding and closure etc. On the other hand, the use of Coffin-Manson also requires some care when applied to the multiaxial elasto/plastic stress field induced around the tip of a notch (even if the global stress field is uniaxial). In short, the apparently more accurate procedures may be sometimes more complicated but basically remain extrapolations, and hence their degree of accuracy may not be necessarily satisfactory.

At the other extreme, i.e. at very low number of cycles (or indeed static failure), rupture is expected to be dominated by plastic flow or brittle fracture, and it is only apparently easier to make an estimate of the strength of a notched component. Most often, in design rules of a notched components, a distinction is drawn between "brittle" and "ductile" materials: in the former case, it is suggested that the peak stress criterion is appropriate, whereas in the second case it is generally considered that a "redistribution" of stresses occurs, such that the ultimate limit is only reached when an entire section of the specimen is loaded at the yield strength. It is pointed out here, however, that the distinction is far from quantitatively clear, as it is well known in the context of fracture mechanics: indeed, a cracked specimen is "brittle" for sufficiently large crack sizes in the sense that it fails by critical condition for propagation of the crack, whereas it would be ductile, i.e. failing by plastic collapse, for lower sizes of the crack, and this independently on the material itself. Hence, the definition of brittleness depends on absolute dimension of the crack, and indeed various authors have recognized this [2-3]. A similar "size effect" is expected therefore to occur for a notch of sufficiently "sharp" geometry and indeed for a rounded notch, although the transition brittle to ductile (and vice-versa) would occur at different geometrical sizes. In the corresponding case of infinite life in fatigue, the equivalent of the transition between ductile and brittle behaviour is the transition between fatigue-limit dominated failure (initiation), and fatigue threshold dominated initiation. Also, it is well recognized in fatigue that notches behave more or less like cracks (crack-like notches, in the Smith and Miller [4] classification and as recognized in the Atzori and Lazzarin [5] criterion) up to a certain size (which depends on material properties), and it is expected that, although plasticity makes a difference, in the static case something similar could happen. Certainly, there is no fundamental reason to make a distinction between "brittle" and "ductile" materials when examining notched structures. In particular, for a very small notch, it is evident that the nominal strength will be unaffected, and would for example remain the nominal yield stress over the net section (or, without any significant difference, the gross, given it is nearly the same) of the specimen, and for larger notch sizes of sufficiently sharp appearance, the effect of the notch will be close to the effect of a crack of "same size", and finally, when the notch size is large enough, the peak stress criterion will become actually the most stringiest condition of all. Notice that the full yield in the net section limit is independent on geometry, so is actually valid also for the crack. Finally, notice that in terms of the gross section, this reasoning translates without modification, when the ratio $A_{\text {net }} / A_{\text {gross }}$, where $A_{\text {net }}$ is the net section, and $A_{\text {gross }}$ is the gross section, is introduced. 
Another reason why notch size effects are less clear than crack size effects, and have perhaps escaped the attention of researchers, is that a notch doesn't have a well-defined "dimension". However, this problem should not be amplified. In fact, the dimension of a crack is only apparently better defined, because this is only when in the case of a through-crack, but the situation is largely less clear when the crack is curved, or has a fully developed $3 \mathrm{~d}$ shape. In this case, as well as in any notch case, the size effect refers in principle to the comparison of specimen of exactly the same geometry (including the crack or the notch features) but of various magnifications. The choice of the linear dimension for the magnification factor is arbitrary. The difficulty starts when the equivalent condition for the comparison with the appropriate material property has to be chosen. This is where the crack and the notch case, strictly speaking, differ. The crack case presents a singular solution which by definition is self-similar, and therefore the entire solution, asymptotically, is given for a certain mode of deformation, independently on the exact geometry. Therefore, a single factor is sufficient to define the "strength" of the singular field - and this the well-known stress intensity factor. In the case of the notch, in principle every geometry is different, in the sense that the stress field cannot be characterized by a single geometrical factor, but in the region where the notch is equivalent to a crack (crack-like notch), the required procedure is to find the path of the equivalent "crack" --although this may not be necessarily simple and uniquely defined. A very important paper by Atzori et al [6] has suggested a criterion to correlate notch and crack behaviour at the fatigue limit to real components and verified by means of an impressive set of 78 fatigue test series for 10 different steels and aluminium alloys taken from the literature. This paper however is limited to the behaviour at the fatigue limit, while here we try to investigate more general trends.

This paper is clearly qualitative, and oriented towards the classical approaches which suggest to interpolate between static strength and fatigue limit: we added to this only the El Haddad type of law, which take into account of Linear Elastic Fracture Mechanics either at the static strength, or at the fatigue limit. However, we haven't added consideration of crack propagation laws, nor compared to these predictions, which so far we are unable to do. Paris law for crack growth has a series of limitations, to cover also the case of "initiation" and "short crack" propagation. For example, in previous studies [7-10], we have shown that for Paris' law to be compatible with the behaviour of SN curves for uncracked materials, one needs to modify it to include also the "material properties" of Basquin law. It would be a final goal to have a model containing the two constants of Paris law and the 2 constants of Basquin law: also, with the two fatigue "thresholds" --- this complete and comprehensive model would amount to six constants in total. However, such a simple model at present is too difficult and not existing. When Paris law constant $\mathrm{m}$ is low, the $\mathrm{SN}$ curve for the material (when uncracked) has a rather different slope than the integrated form of Paris law [11-12], that is $\mathrm{k}>>\mathrm{m}$. Possible corrections would need to include the effect of plasticity at the crack tip, which effectively increases the size of the "equivalent crack", but again this is not pursued in the present paper.

The scope of the present paper is therefore to try to "unify" crudely various concepts for static and fatigue design, without any intention to give radically new methodologies, or empirical formulae, but with the simpler scope of examining various ranges of validity and overlap between the theories which often are treated separately, and with principally the suggestion to use interpolation between robust estimates of limit conditions and the use of all the material properties which are available, rather than extrapolation from a single methodology using a limited set of material properties, independently on how refined the methodology may appear to be. This is not necessarily limited to preliminary calculations, but also when there is possibility of some experimental investigations, as a simpler route for understanding of the behaviour in fatigue of a notched component. Ultimately, the core of the message becomes quite obvious to the engineer, and indeed it is the base of various standard procedures for specific fields, like for example the design guides of gears (see for example [13]): "interpolate" between limit conditions, using some knowledge of the notch size effect (in the lack of direct experimental data) as recently emerged more clearly at least for the infinite life region. In particular, the entire spectrum of possible behaviour can be described in a single diagram strength vs. notch/crack size.

\section{EMPIRICAL LAWS IN FATIGUE}

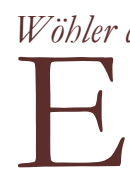

\section{urve}

mpirical laws have emerged in fatigue since when Wöhler was conducting his famous experiments of rotating bending fatigue in railways axles for the German State Railways in the 1860s. Various authors noticed empirically that it was convenient to plot SN data on a $\log / \log$ (or a semi-log) diagram (for a detailed study of the old literature see the recent paper by Sendeckyj [14]). Since then, the so-called Wöhler SN diagram has been widely used. There is no fundamental reason to write the curve as a power-law, and indeed alternative equations have been suggested, but the power law between 2 given points is probably the simplest or most used form for the plain specimen, in the form (see Fig.1): 


$$
N_{0} \sigma_{R}^{k}=N_{\infty} \Delta \sigma_{0}^{k}=N_{f} \Delta \sigma, \quad N_{0}<N_{f}<N_{\infty}
$$

where $\Delta \sigma$ is the stress range (we assume at the moment for simplicity that amplitude and range coincide i.e. the load ratio $\mathrm{R}=0$, although it is clear that in general it would perhaps be appropriate to rewrite Eq.(1) in terms of amplitude of the cycle $\sigma$ ) and the $N_{0}$, and $N_{\infty}$ are the number of cycles as defined in Fig.1. Clearly, Eq.1 also implies

$$
k \log \left(F_{r}\right)=k \log \left(\frac{N_{\infty}}{N_{0}}\right)
$$

and typically for steels considering $N_{\infty}=10^{7}$ and $N_{0}=10^{3}$, for $F_{R}=\sigma_{R} / \Delta \sigma_{0}=2$ we would have $k=13.3$, while for $F_{R}=3, k=8.4$, in the typical range $k=6-14$ for $\mathrm{Al}$ or ferrous alloys.

In strain-controlled fatigue, the fatigue curve is replaced by a sum of two power/law functions assuming the fatigue life to be dominated by plastic strain in the LCF regime, and elastic strains or stresses in the HCF. The resulting well know equation (Coffin/Manson) is expected to be more accurate (if anything because it has more degrees of freedom to reproduce the experimental SN curve) although there is still a need to introduce the cut-off thresholds on very low and very high number of cycles, particularly on the low number of cycles where it tends to have the wrong concavity.

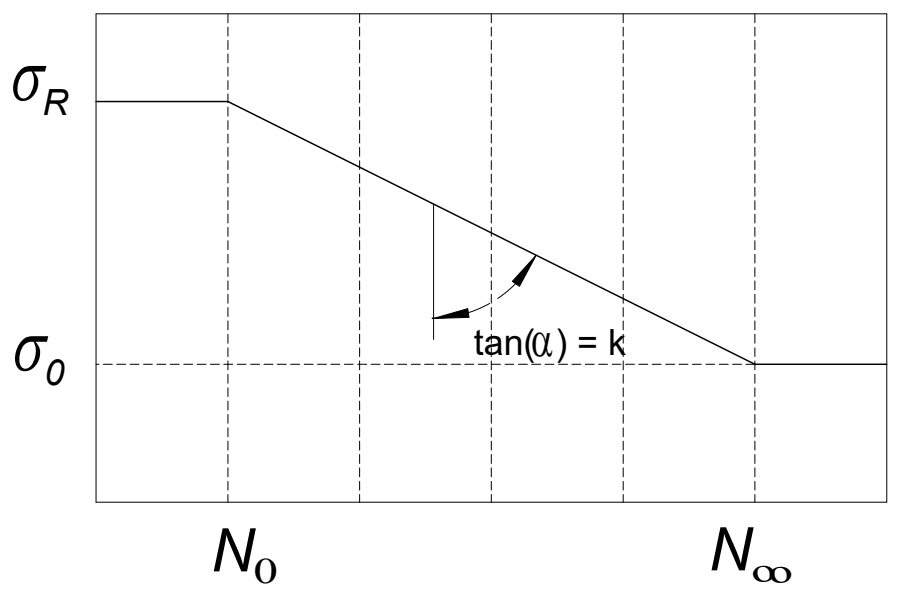

Figure 1: The simplified Wohler curve.

\section{Paris' law}

The second important power law in fatigue is Paris' law, giving the advancement of fatigue crack per cycle, $v_{a}$, as a function of the amplitude of stress intensity factor $\Delta \mathrm{K}$ (see Fig.2)

$$
v_{a}=\frac{d a}{d N}=C \Delta K^{m} ; \quad \Delta K_{t h}<\Delta K<K_{I c}
$$

where $\Delta K_{t b}$ is the "fatigue threshold", and $K_{I c}$ the "fracture toughness" of the material. There is therefore no dependence on absolute dimension of the crack. The law is mostly valid in the range $10^{-5}-10^{-3} \mathrm{~mm} / \mathrm{cycle}$, and in a simplified form it can be considered intersecting $\Delta K_{\text {th }}$ and $K_{I c}$ at $10^{-6}, 10^{-4} \mathrm{~mm} / \mathrm{cycle}$, respectively. This means that the constant $\mathrm{C}$ is not really arbitrary, since by writing the condition at the intersections, $C=\frac{10^{-6}}{\Delta K_{t h}^{m}}=\frac{10^{-4}}{K_{I c}^{m}}$.

An alternative form can be obtained considering that Paris' law is in general valid in the range $10^{-5}-10^{-3} \mathrm{~mm} /$ cycle and hence instead of the constant $C$ it is perhaps more elegant to define a constant $\Delta K_{4}$, i.e. the range corresponding to a speed of propagation of $10^{-4} \mathrm{~mm} /$ cycle 


$$
v_{a}=C^{\prime}\left(\frac{\Delta K}{\Delta K_{-4}}\right)^{m}
$$

where $C^{\prime}=10^{-4} \mathrm{~mm} /$ cycle by definition. In other words,

$$
C=C^{\prime}\left(\frac{1}{\Delta K_{-4}}\right)^{m}
$$

From the linearity in this range $10^{-5}-10^{-3} \mathrm{~mm} / \mathrm{cycle}$ in the $\log / \log$ plot, Fleck et al [1] suggest to find the Paris exponent $m$ as

$$
\log F_{k}=\frac{4}{m}
$$

and Fig.16 of their paper seems to confirm this assumption. More in general, it is possible to assume

$$
m \log F_{k}=\log \frac{v_{a}^{c}}{v_{a}^{t h}}
$$

where $v_{a}^{\text {th }}$ is a conventional velocity at the threshold, and $v_{a}^{c}$ at the critical conditions.

A first obvious (and well known) link between the two curves (Wöhler and Paris) is obtained when considering the life of a distinctly cracked specimen having an initial crack size $a_{i}$. Under the assumptions of constant remote stress and no geometrical effects, for $m>2$ the following is obtained (where the dependence on the final size of the crack $a_{f}$ has been removed as relatively not influent)

$$
a_{i}^{\frac{(2-m)}{m}}=\left(\frac{m-2}{2}\right) C \pi^{m / 2} \Delta \sigma^{m} N_{f}
$$

This is to be considered as a Wöhler curve of the cracked component and the Wöhler exponent turns out to be exactly equal to the Paris exponent, $k^{\prime}=m$. It is interesting however to remark that the SN curve depends on the initial crack size, $a_{i}$. Hence the threshold condition from Eq. (8) would tend not to coincide with that directly obtained from the threshold value which also depends on $a_{i}$ but with a different power

$$
\Delta \sigma_{\text {lim }, t h}=\frac{\Delta K_{t b}}{\sqrt{\pi a_{i}}}
$$

In fact the two powers in Eqs. $(8,9)$ coincide only if $(m-2) / 2 m=1 / 2$ which is only true for very high $m$, showing in fact that the Paris law should near the threshold have a vertical continuous slope, and the simplification of the Paris law corresponds to a bifurcation to the solution given by the two branches (the threshold, and the power-law regime). This is another example of the risk of using these equations for extrapolations, without considering also the other information we have on the material properties.

So far, we have only dealt with the case of either completely uncracked or the distinctly cracked specimen. Most real cases would include notched specimen, or cracks of small size. We therefore need to introduce the theories on the effect of notches and cracks of varying size on fatigue life.

\section{Kitagawa and Atrori/Lazzarin diagrams}

For infinite life (or safe-life) design, Atzori \& Lazzarin [5] have recently proposed a new diagram (a generalization of the celebrated Kitagawa diagram), which serves as a single "map" showing the fatigue limit reduction due to notch and cracks as a function of defect (or notch) size. For the interaction between fatigue limit and fatigue threshold for short cracks in the 
Kitagawa diagram El Haddad et al. [15] had proposed the famous interpolating equation (concept of defect sensitivity): for a centred crack of size $a$, in terms of failure for a range $\Delta \sigma_{f}$

$$
\Delta \sigma_{f}=\frac{\Delta K_{t h}}{\sqrt{\pi\left(a+a_{0}\right)}}
$$

where $a_{0}$ is the intrinsic material size for infinite life, defined as

$$
a_{0}=\frac{1}{\pi}\left(\frac{\Delta K_{t h}}{\Delta \sigma_{0}}\right)^{2}
$$

where $\Delta \sigma_{0}$ is fatigue limit and $\Delta K_{t b}$ is fatigue threshold of the material. In fact it is well known that cracks smaller than this size do not follow Paris law not even for $\Delta K>\Delta K_{t b}$, whereas the material is limited in this range by the fatigue limit, $\Delta \sigma_{0}$.

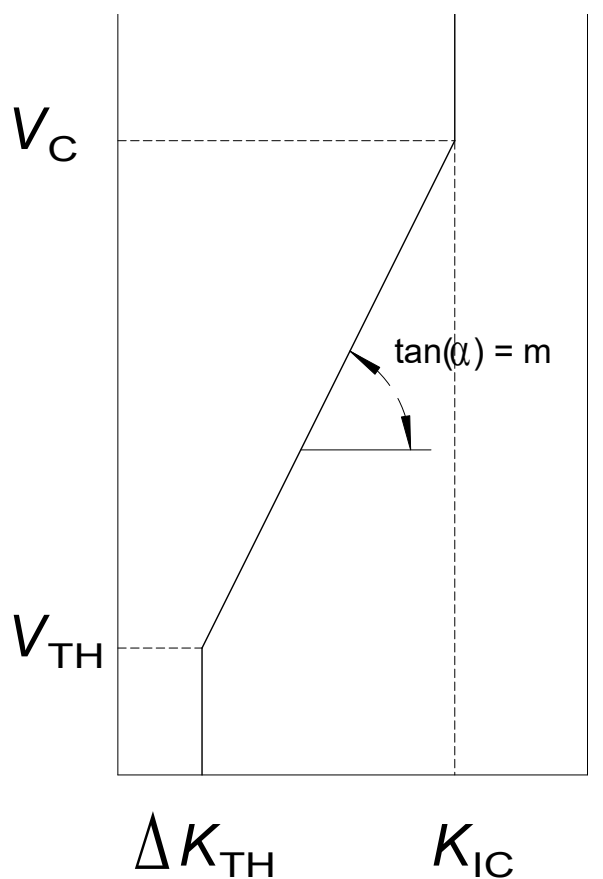

Figure 2: The Paris law.

The denomination "intrinsic crack" is due to the fact that the fatigue limit from (11) is also

$$
\Delta \sigma_{0}=\frac{\Delta K_{t h}}{\sqrt{\left.\pi a_{0}\right)}}
$$

and hence (10) is equivalent to (12) when the intrinsic crack is added. As originally proposed by Smith \& Miller [4] any notch is practically equivalent to a crack up to a certain size, depending on the stress concentration factor, $K_{t}$. Hence, Atzori \& Lazzarin [5] suggested to consider only (i) crack-like behaviour treatable with standard fracture mechanics (in particular, with Eq.(10)) and (ii) large blunt notches only, treatable with the simplest stress concentration factor approach. This is exemplified in the lines of Fig.3. For a constant size of the notch, this criterion can also be put in terms of a limit $K_{t}, K_{t}^{*}$, beyond which fatigue limit is no further decreased, giving an area where cracks are supposed to initiate from the notch but not propagate, the so-called "non-propagating crack zone". Notches with $K_{t}>K_{t}^{*}$ behave as defects of same dimension, i.e. are "crack-like 
notches". By defining instead of a transitional stress concentration factor, as transitional size of the notch, $a^{*}$, as the intersection of the horizontal line $\Delta \sigma_{0} / K_{t}$ with the long crack threshold, gives ${ }^{1}$

$$
\frac{a^{*}}{a_{0}}=K_{t}^{2}
$$

For notches lager than this size $\mathrm{a}^{*}$, simply the peak stress condition can be written in terms of failure range $\Delta \sigma_{f}$

$$
\Delta \sigma_{f}=\Delta \sigma_{0} / K_{t}
$$

where $K_{t}$ is the stress concentration factor.

It is natural to extend these concepts to the static failure case, drawing an El-Haddad "equivalent line" for the static case, and accordingly introduce the dimensions $a_{0}^{S}$ analogous to (11) and depending this time by $K_{I c}$, the toughness of the material and $\sigma_{R}$ its tensile strength as

$$
a_{0}^{s}=\frac{1}{\pi}\left(\frac{K_{I c}}{\sigma_{R}}\right)^{2}
$$

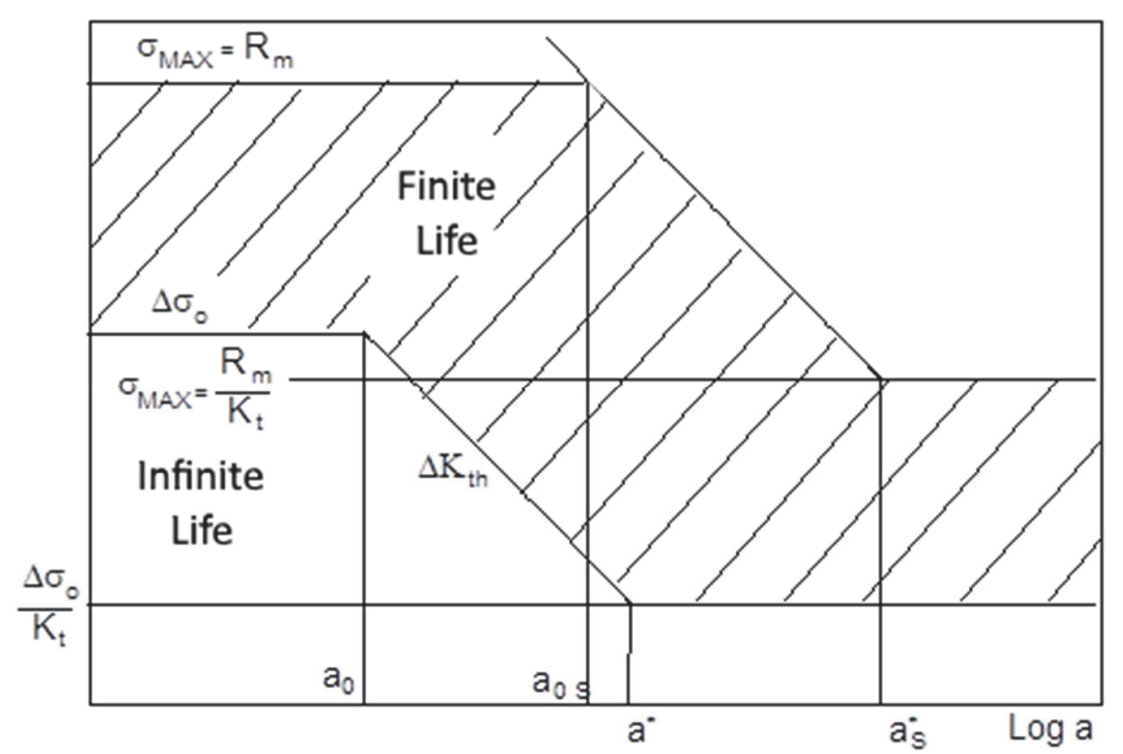

Figure 3: The Atzori-Lazzarin generalized diagram (Atzori \& Lazzarin [16]).

\section{FATIGUE AND CRACK “SENSITIVITIES" AND OTHER MATERIAL PROPERTIES}

I

$\mathrm{n}$ Fleck et al [1] and in Ashby [17, 18], a large number of material properties of interest are given, and of particular interest are the "intrinsic crack" sizes, $\mathrm{a}_{0}$, and $\mathrm{a}_{0} \mathrm{~s}$ which can be retrieved qualitatively from some of the maps. They permit to classify "crack sensitivity" of the material, under static and fatigue load respectively (for example, a material

\footnotetext{
${ }^{1}$ More precisely, the intersection should be defined with the El Haddad line not the long crack threshold. The difference can be neglected however, if the stress concentration is not too small.
} 
with high $\mathrm{a}_{0}$ will tend to be insensitive to cracks up to the size of the order of $\mathrm{a}_{0}$ in fatigue). Analogously, the two ratios $F_{K}=\frac{K_{I c}}{\Delta K_{t h}}$, and $F_{R}=\frac{\sigma_{R}}{\Delta \sigma_{0}}$ define the "fatigue sensitivities".

Specifically, materials with high $F_{K}$ are fatigue sensitive when cracked, and those having large $F_{R}$ are fatigue sensitive when uncracked. In the former case, in the presence of a crack it is useful to design for fatigue crack propagation (like in the "damage tolerance" design approach), because the static limit is very high and the threshold condition is perhaps too strict, and there is margin to gain from a more elaborate design. Similarly, when $F_{R}>>1$, it is convenient to design when uncracked for the fatigue limit, or perhaps to the finite life required. The opposite is true when $F_{K}, F_{R}$ are both small and close to one, in which case it is generally sufficient to design statically. Finally, notice that as generally $F_{K}>>F_{R}$, a material sensitive to fatigue when uncracked is likely to be also sensitive to fatigue when cracked, whereas the vice versa is not true, a material sensitive to fatigue when cracked may not be sensitive to fatigue when uncracked.

The two sensitivities ("crack sensitivity" and "fatigue sensitivity") are not unrelated, as obviously $a_{0} s / a_{0}=\left(F_{K} / F_{R}\right)^{2}$ : when $F_{K}>>F_{R}$ as it is usual, $a_{0} s / a_{0}>>1$ a fortiori. In other words, a material that is more sensitive to fatigue when uncracked than when cracked, then in terms of tolerance to crack sizes, is significantly more sensitive to cracks in fatigue than in static loading. Materials which are equally sensitive to fatigue when cracked or uncracked, would have equal sensitivity to cracks under fatigue or static loads.

From the maps in Fleck et al [1] and in Ashby [17, 18], a large number of qualitative data can be retrieved on these material properties and their ratios, as well as the characteristic sizes $a_{0}, a_{0}^{S}$ (which in turn for a given stress concentration factor can be put in terms of $a^{*}, a^{* S}$ ). For example, two maps are reproduced in Fig.4,5 here. In particular, Fig.4 gives the fatigue threshold vs the fatigue limit (in terms of amplitude endurance limit), and constant lines of $\frac{1}{4 \pi}\left(\frac{\Delta K_{t h}}{\sigma_{e}}\right)^{2}$, which can be put in relationship with the $a_{0}$ defined in (11). For $a_{0}$ we recognize values around $1 \mu \mathrm{m}$ for some ceramic materials, up to few $\mathrm{mm}$ for some metallic alloys or polymers), whereas for the corresponding $a_{0}^{S}$ we see the value for composite materials, whereas in this particular collection for metals and polymers the yield stress rather than the failure stress is given and hence the plastic radius can be estimated rather than our $a_{0}^{S}$, and finally for rocks and ceramics the compression failure stress is given. In all cases, we notice a certain correlation i.e. grouping around the diagonal line, corresponding to a tendency to have high values for properties at same time (however, within this general trend, there are remarkable exceptions, especially within single class of materials). However, it is seen that this holds more for uncracked properties, i.e. $F_{R}$ is relatively constant for materials (and for the definition of $F_{R}$ in Fleck et al [1] and in Ashby [17, 18] for some metals and polymers, we find $F_{R}>1$ ). Vice versa, $F_{K}$ varies significantly more and more still $a_{0}^{S}$ and $a_{0}$ (particularly $a_{0}^{S}$ ). In other words, as it is commonly known, to an increase of strength does correspond generally an increase of fatigue strength, but an increase of toughness does not always correspond to an increase of threshold. Moreover, to a greater threshold not always corresponds an increased fatigue limit, and even more the case that to an increase of toughness corresponds an increase of static strength. For example, for steel and metallic alloys, as is well known, to greater yield strength corresponds a reduced toughness, but this is not true for other classes of materials, such as composites, ceramics and cements.

In general, $F_{k}>>F_{R}$, and for metals typical values are 5-20, and 2, respectively, so that $a_{0}^{S}$ is about 100 times greater than $a_{0}$.

\section{GENERAL WÖHLER CURVE}

7 he two Atzori-Lazzarin curves (static, and infinite life) permit some qualitative considerations on the intermediate, finite life, region of notched and cracked structures. The resulting map for general cracked or notched specimen is as shown in Fig.3, as first presented by Atzori-Lazzarin at a conference in Italy [16]. The shaded area corresponds to the "finite life region". Given a material with Wöhler curve of exponent $k$, and of Paris exponent $m$, we expect that the limiting Wöhler curve exponent for a notched component will be the one for a large crack obeying Paris' law, for which $k_{\text {lim }}=m$. Therefore, we can expect a notch of varying size and sharpness to cause a reduction of the Wöhler slope $k<k^{\prime}(a)<m$, i.e. intermediate between $k$ and $m$. Some calculations show this is indeed the case, and draw some estimates on the obtained values. 


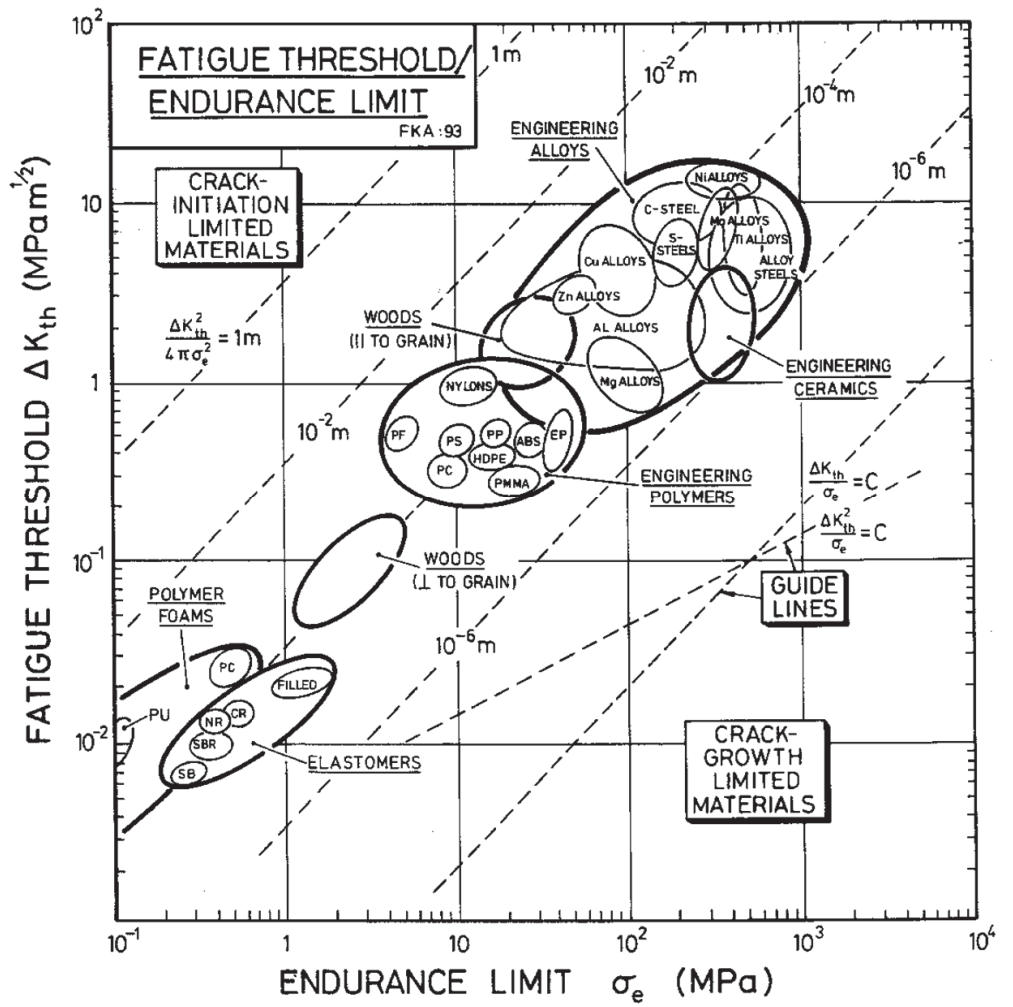

Figure 4: Asbhy maps for fatigue threshold vs. fatigue endurance limit (reproduced with permission from (Fleck et al. [1], Fig. 10).

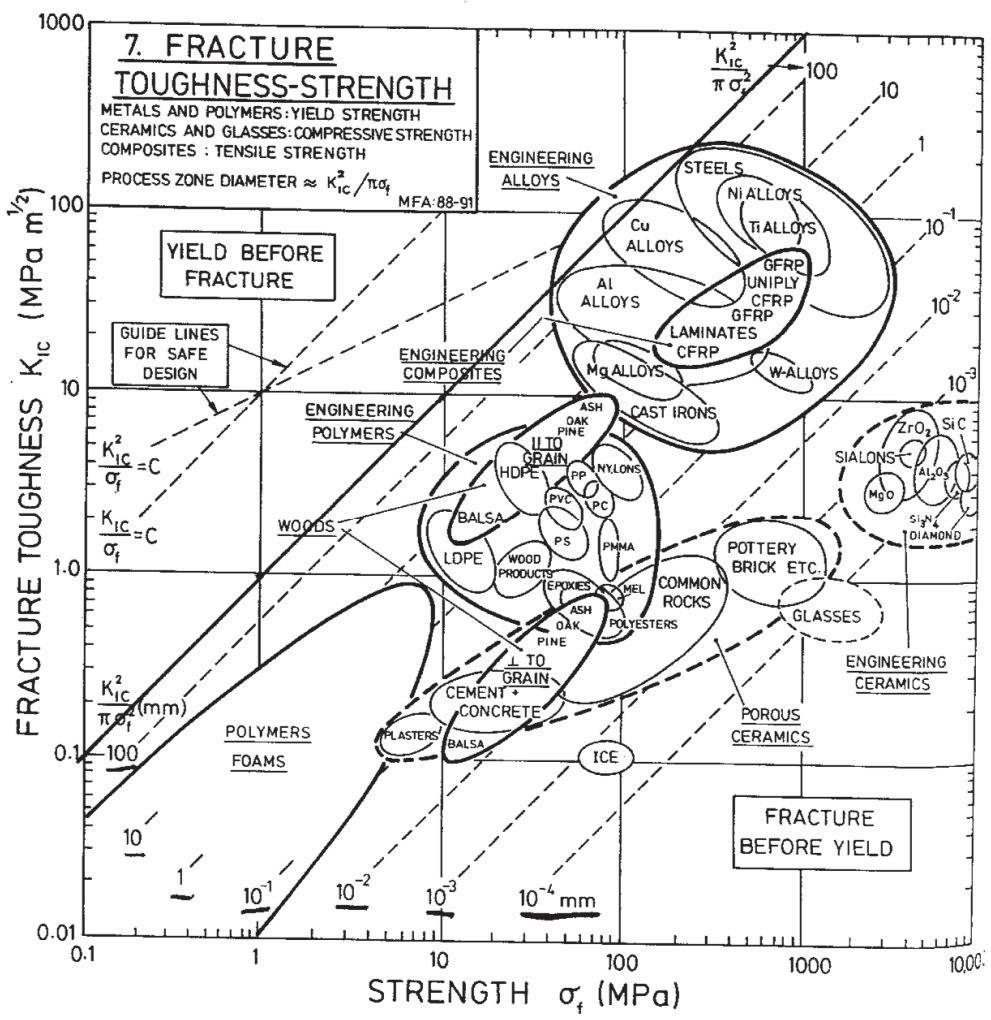

Figure 5: Asbhy maps for fracture thoughness vs. strength (see appropriate definitions in the legend). Reproduced with permission from Asbhy [17], Fig. 4.8, or Asbhy [18], Fig.10). The contour lines indicate the size of plastic radius. 
By looking at fixed dimension of the notch, and interpolating between static and infinite-life strength, we can obtain the "generalized Wöhler curve", as well as a "generalized Wöhler coefficient, $k$ ' $(a)$ ". We shall start with a simplified version of the Atzori-Lazzarin schematically represented in Fig.5, i.e. the criterion with line segments, to make the easiest possible estimate of the generalized slope $k^{\prime}---$ we shall return to the more accurate El Haddad version in the last paragraph. Since $a_{0}^{s} / a_{0}=\left(\mathrm{F}_{K} / \mathrm{F}_{\mathrm{R}}\right)^{2}$ and $a^{*} / a_{0}=\mathrm{K}_{\mathrm{t}}^{2}$ we also obtain $a_{0}^{S} / a^{*}=\left(\mathrm{F}_{K} / F_{\mathrm{R}}\right)^{2} / \mathrm{K}_{\mathrm{t}}^{2}$. Hence if $\mathrm{K}_{\mathrm{t}}<\mathrm{F}_{\mathrm{K}} / \mathrm{F}_{\mathrm{R}}$ then $a_{0}{ }^{S}>a^{*}$ whereas if $\mathrm{K}_{\mathrm{t}}$ $>\mathrm{F}_{\mathrm{K}} / \mathrm{F}_{\mathrm{R}}$ then $a_{0}^{s}<a^{*}$. We shall only consider $\mathrm{F}_{\mathrm{K}}>\mathrm{F}_{\mathrm{R}}$ or as a limit case, $\mathrm{F}_{\mathrm{K}}=\mathrm{F}_{\mathrm{R}}$ hence we have 3 cases:

1. Case (a) $\mathrm{F}_{\mathrm{K}}>\mathrm{F}_{\mathrm{R}}$ and $\mathrm{K}_{\mathrm{t}}<\mathrm{F}_{\mathrm{K}} / \mathrm{F}_{\mathrm{R}}$ (top of Fig.6 where we see $a_{0}<a^{*}<a_{0}{ }^{5}<a s^{*}$ )

2. Case (b) $\mathrm{F}_{\mathrm{K}}=\mathrm{F}_{\mathrm{R}}$ and $\mathrm{K}_{\mathrm{t}}>\mathrm{F}_{\mathrm{K}} / \mathrm{F}_{\mathrm{R}}=1$ (bottom of Fig.6 where we see $a_{0}=a_{0}{ }^{s}<a^{*}=a s^{*}$ )

3. Case (c) $\mathrm{F}_{\mathrm{K}}>\mathrm{F}_{\mathrm{R}}$ but $\mathrm{K}_{\mathrm{t}}>\mathrm{F}_{\mathrm{K}} / \mathrm{F}_{\mathrm{R}}$ (Fig.7 where we see $\left.a_{0}<a_{0}{ }^{s}<a^{*}<a_{s}^{*}\right)$

In the original case of Fig. 5 we recognize case (b) which is also typical for metals for high stress concentrations whereas also case (a) is possible since $\mathrm{F}_{\mathrm{K}} / \mathrm{F}_{\mathrm{R}}=2-10$. In any event, 5 regions can be seen in the Atzori-Lazzarin diagram. Fig.6 also show the construction of the generalized Wöhler curves corresponding to the key sizes (in other words, we have up to 4 distinct Wöhler lines corresponding to the sizes $\left.a_{0}, a_{0}{ }^{5}, a^{*}, a_{s}^{*}\right)$.

With reference to Fig.5 (also reproduced in the details of the construction in Fig.7), the 5 regions correspond to different trend of the resulting slope in the $\left(a_{0}<a_{0} s<a^{*}<a^{*}\right)$. In the first region, for $a<a_{0}, k^{\prime}=k$ remains unvaried to the value of the unnotched specimen. In the second region $a_{0}<a<a 0^{s}$, we obtain a decrease of $k^{\prime}$ 'up to a limit value, then remaining constant in the entire third region $a_{0}{ }^{S}<a<a^{*}$, and which we obtain easily from writing a Wohler-like power-law between the static strength value to the fatigue limit divided by the $\mathrm{K}_{\mathrm{t}}$ factor,

$$
N_{0} \sigma_{R}^{k^{\prime}}=N_{\infty}\left(\frac{\Delta \sigma_{0}}{K_{t}}\right)^{k^{\prime}}
$$

If we divide the original Wöhler curve (1) by (16) term by term, we obtain

$$
\frac{\sigma_{R}^{k}}{\sigma_{R}^{k^{\prime}}}=N_{\infty} \frac{\Delta \sigma_{0}^{k}}{\Delta \sigma_{0}^{k^{\prime}}} K_{t}^{k^{\prime}}
$$

i.e.

$$
\left(k^{\prime}\right)_{\min }=\frac{k \log \left(F_{R}\right)}{\log \left(K_{t} F_{R}\right)}
$$

In other words, $k$ ' decreases from the unnotched specimen case up to a limit value (depending on Kt) given by Eq.18. Notice that this equation has been obtained without any need to specify $N_{0}$ and $N_{\infty}$, except of course that these values are assumed to remain constant independently on the size of the notch. If a more general choice had been made, i.e. using new values $N_{0}^{\prime}$ and $N_{\infty}^{\prime}$, and not the original $N_{0}^{\prime}$ and $N_{\infty}^{\prime}$ of the Wöhler curve in Eq.1,

$$
N_{0} \sigma_{R}^{k^{\prime}}=N_{\infty}^{\prime}\left(\frac{\Delta \sigma_{0}}{K_{f}}\right)^{k^{\prime}}
$$

and dividing again for (1)

$$
\left(k^{\prime}\right)_{\text {min }}=\frac{k \log \left(F_{R}\right)-\log \left(\frac{N_{\infty} / N_{0}}{N_{\infty}^{\prime} / N_{0}^{\prime}}\right)}{\log \left(K_{t} F_{R}\right)}
$$

and the decrease of $k^{\prime}$ depends now on the variation of $N^{\prime} 0$ and $N^{\prime}$, as a function of the notch size, and not just on $K_{t}$. However, we shall neglect this possibility. 
The limit case is when the stress concentration is high enough that $K_{t}>F_{K} / F_{R}$ in which case the limit ratios is obtained between the static and the fatigue limits, and consequently from (18)

$$
\left(k^{\prime}\right)_{\lim }=\frac{k \log \left(F_{R}\right)}{\log \left(F_{K}\right)}
$$

which is clearly the highest slope compatible to our criteria and the material properties ratios. The more general equation analogous to Eq. (20) could be obtained by using $K_{t}>F_{K} / F_{R}$ in (20) or combining eqt(21) with Eq. (6-7), obtaining in any case

$$
\left(k^{\prime}\right)_{\lim }=m \frac{\log \left(\frac{N_{\infty}}{N_{0}}\right)}{\log \left(\frac{v_{a}^{c}}{v_{a}^{t h}}\right)}
$$

which clearly seems to link the limit generalized Wöhler slope to the Paris slope and the position of the key points in the Wöhler and Paris laws, as it is correct since the limit generalized Wöhler slope is indeed significant in the region where life would be mainly given by propagation. In fact, turning back to the standard assumptions for the key points $\left(v_{a}^{\text {th }}, v_{a}^{c}=10^{-6}\right.$, $10^{-2} \mathrm{~mm} /$ cycle and, perhaps with less generality, $N_{\infty}=10^{7}$ and $N_{0}=10^{3}$ cycles), we re-obtain the comforting result that the limiting Wöhler coefficient coincides numerically with the Paris coefficient:

$$
k_{\text {lim }}^{\prime}=m \frac{(7-3)}{-2+6}=m
$$

as it was obtained independently from integrating Paris' law in (8).

Turning back to our classification, we have finally a $4^{\text {th }}$ region, where $a^{*}<a<a^{*}$. where the slope starts to increase again towards the original value, $k$, which then remains constant in the fifth region. This is not too hard to interpret, given that a very large blunt notch basically behaves as a standard specimen subject to a nominal stress $K_{t}$-times higher than the remote stress. In other words, we suggest that we start from the basic Wöbler curve behaviour, we move towards Paris with increasing notch size, but we then return to the original Wöbler behaviour again, for very large blunt notches. Notice that only the Wöhler curves corresponding to the key sizes are reproduced in Fig.6, whereas also some intermediate ones are included in Fig.7 with lighter line.

For Case (a), $F_{K}>F_{R}$ but $K_{t}<F_{K} / F_{R}$ the limiting slope is not reached, and we only obtain the minimum slope as (18) or (20). We then decrease slope in the fourth region and return to the original one in the fifth.

The case (b) is somehow contrived and would correspond to an abrupt transition from Wöbler towards Paris and back to Wöbler, the abrupt transition being particularly evident because of the schematic form of the criterion.

\section{WÖHLER CURVES USING El HADDAD}

$\mathrm{I}$

$\mathrm{n}$ the previous paragraph, we used the schematic version of the static and infinite life Atzori-Lazzarin criteria. Here, we shall use the El Haddad Eqs. (10) and the corresponding static case. Notice we can put these two equations in the form of a reduction fatigue $\mathrm{K}_{\mathrm{f}}$ in fatigue, and the corresponding $\mathrm{K}_{\mathrm{s}}$ reduction static factor

$$
K_{f}=\left(\begin{array}{c}
\sqrt{1+\frac{a}{a_{0}}}, \text { for } a<a^{*} \\
K_{t}, \text { for } a>a^{*}
\end{array}\right)
$$

and 


$$
K_{S}=\left(\begin{array}{c}
\sqrt{1+\frac{a}{a_{0}^{S}}}, \text { for } a<a_{S}^{*} \\
K_{t}, \text { for } a>a_{S}^{*}
\end{array}\right)
$$
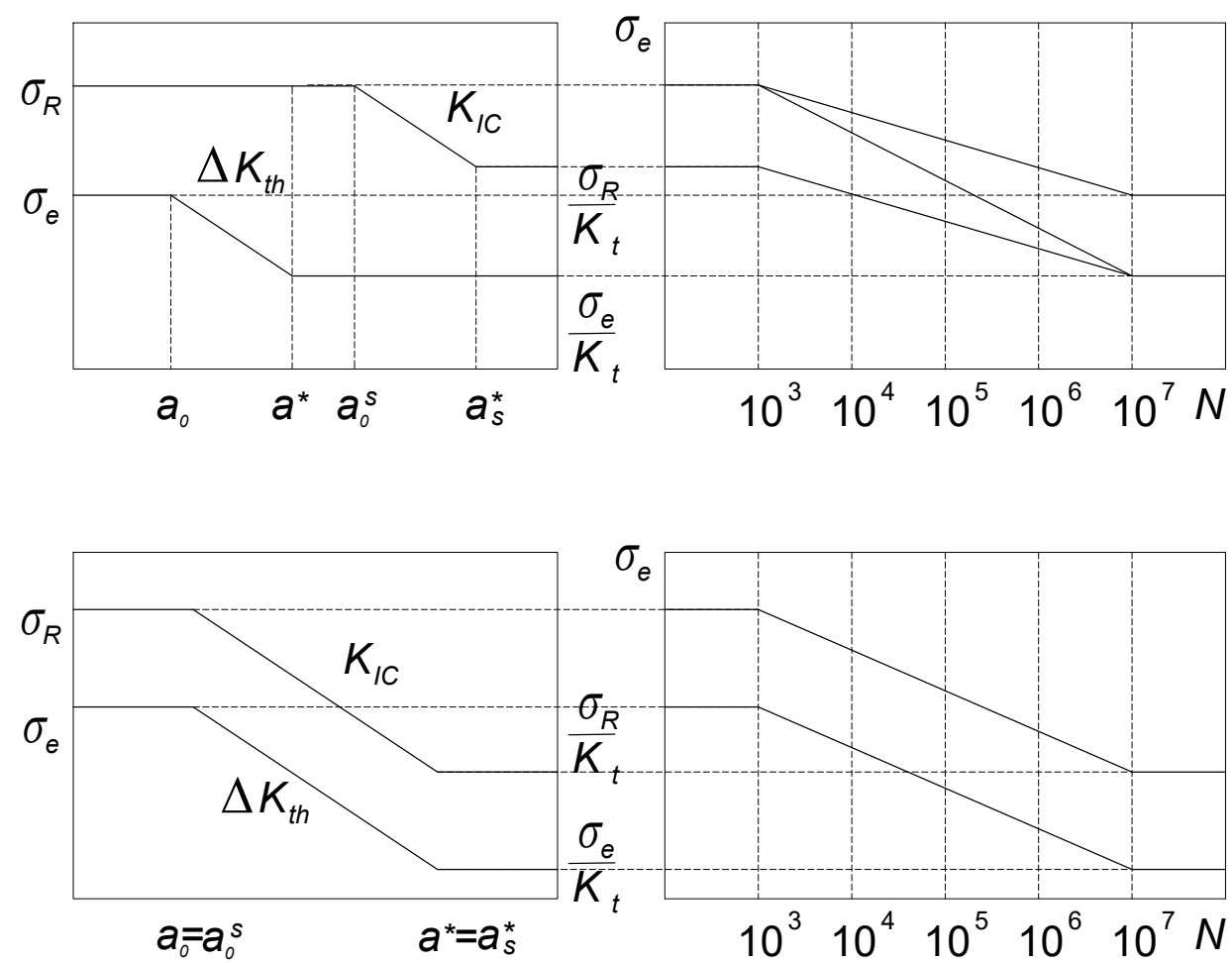

Figure 6: The interpolation procedure: example case (a) $\mathrm{FK}>\mathrm{FR}$ and $\mathrm{Kt}<\mathrm{FK} / \mathrm{FR}$ and case (b) $\mathrm{FK}=\mathrm{FR}$ and $\mathrm{Kt}>\mathrm{FK} / \mathrm{FR}=1$.
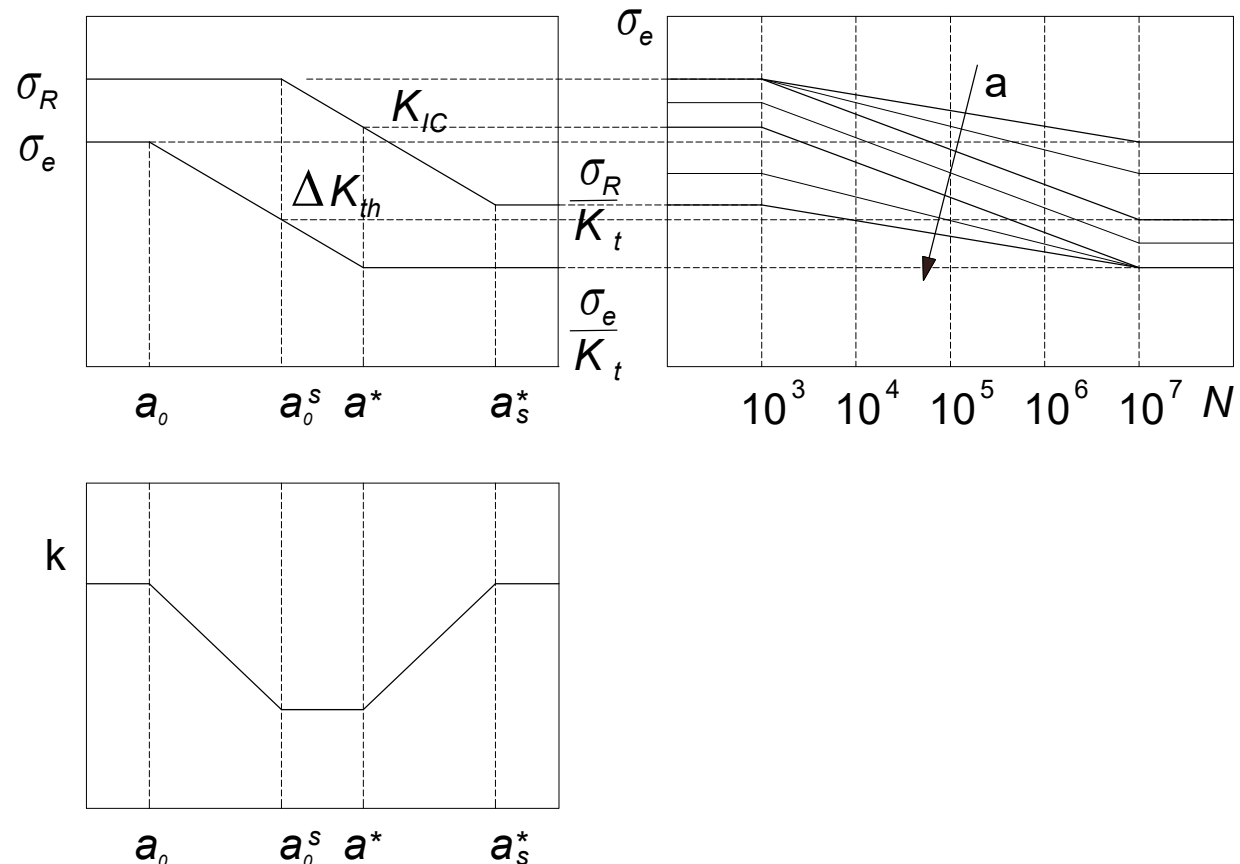

Figure 7: The generalized Wohler slope as it results from an example interpolation procedure. Case (c) FK $>$ FR but Kt $>$ FK/FR. 
These two curves (or better the inverse of these two curves) are reproduced in Fig.8,9, as a function of a/a for some example cases (typical steel and typical ceramic, where $F_{K}=15.5, F_{R}=2.4$, and $F_{K}=2, F_{R}=1.5$, respectively). Since the plots are given as a function of $\mathrm{a} / \mathrm{a}_{0}$, the $1 / \mathrm{K}_{\mathrm{f}}$ curve "bends" around $\mathrm{x}=1$, whereas the corresponding $1 / \mathrm{K}_{\mathrm{S}}$ curve "bends" around $\mathrm{x}=a_{0} s / a_{0}$ which in fact scales with the square of the ratio $F_{K} / F_{R}=15.5 / 2.4=6.5$, and $F_{K}=2 / 1.5=1.33$, and hence $a_{0} s /$ $a_{0}=41.7$ and 1.7 respectively, since $a_{0} s / a_{0}=\left(F_{K} / F_{R}\right)^{2}$.

This El Haddad form is apparently more complicated, but in fact by repeating the same reasoning of the previous paragraph, we only need to distinguish 2 possible ranges: for $a<a^{*}$,

$$
\frac{k^{\prime}}{k}=\frac{\log F_{\mathrm{R}}}{\log \left[\frac{F_{K} \sqrt{a+a_{0}}}{\sqrt{a+a_{0}^{s}}}\right]}
$$

i.e.

$$
\frac{k^{\prime}}{k}=\frac{\log F_{R}}{\log \left[\frac{F_{K} \sqrt{\frac{a}{a_{0}}+1}}{\sqrt{\frac{a}{a_{0}}+\left(\frac{F_{K}}{F_{R}}\right)^{2}}}\right]}
$$

This slope has a limiting value of

$$
\left(\frac{k^{\prime}}{k}\right)_{\lim }=\frac{\log F_{R}}{\log \left[\frac{F_{K} \sqrt{K_{t}^{2}+1}}{\sqrt{K_{t}^{2}+\left(\frac{F_{K}}{F_{R}}\right)^{2}}}\right]} ; \quad a=a^{*}
$$

For $a>a^{*}$, the slope increases again,

$$
\frac{k^{\prime}}{k}=\frac{\log F_{R}}{\log \left[\frac{K_{I c} / \sqrt{\pi\left(a+a_{0}^{s}\right)}}{\Delta \sigma_{0} / K_{t}}\right]}
$$$$
a^{*}<a<a_{s}^{*}
$$

and finally we return to the original slope $k$, for $a>a_{s}^{*}$.

The resulting slopes are also indicated as ratio $k^{\prime} / k^{\prime}<1$ in the Fig.8,9 for 3 example stress concentration factors $K_{t}=2,5,10$, showing how for steel the generalized Wöhler slope is already about $60 \%$ of the original one for notches slightly larger than $a_{0}$ and with stress concentration factor only of about 2 . The slope continues to decrease to about $40 \%$ when the notch is now significantly larger than $a_{0}$ (specifically about 20 times larger than $a_{0}$ ) and recollecting Eq. 6,2 for the estimate of the Paris and Wöhler slopes, respectively, we have about $m=3.4, k=10.5$ with the conclusion that the limit reduction of the generalized $W \ddot{\partial h l e r}$ slope is $k^{\prime} / k=32 \%$, and hence with a stress concentration factor of about 5 we're already very close to the limit slope.

For the case of ceramic material in Fig.9, the estimates with eqts. 6,2 give $m=13.3$ and $k=22.7$ with the conclusion that the limit reduction of the generalized Wöbler slope is $59 \%$. However, with the same concentration factors as the previous cases, i.e. $K_{t}=2,5,10$ we obtain that the decrease of the slope is already almost complete with a notch of the order of $2 a_{0}$ and with 
the smallest $K_{t}=2$. In this respect, the brittle ceramic material is more sensitive to small notches, and this is not entirely a surprise.

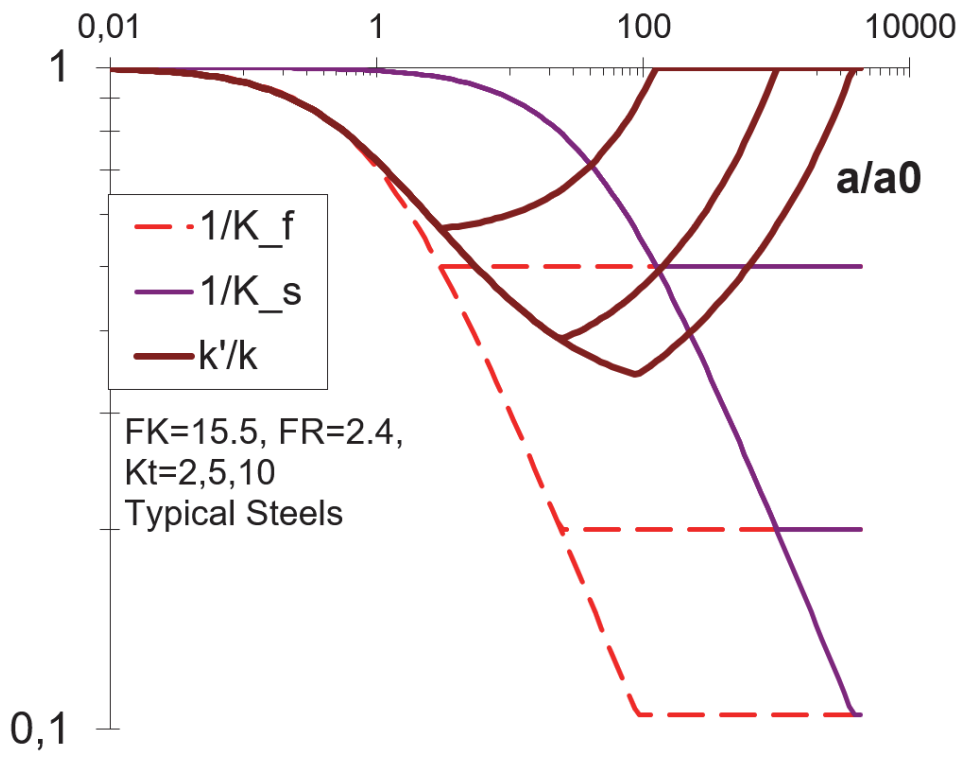

Figure 8: The generalized Wohler slope as it results from an interpolation procedure using the El Haddad equation for both the static and the fatigue criteria, and for typical material constant ratios of steels.

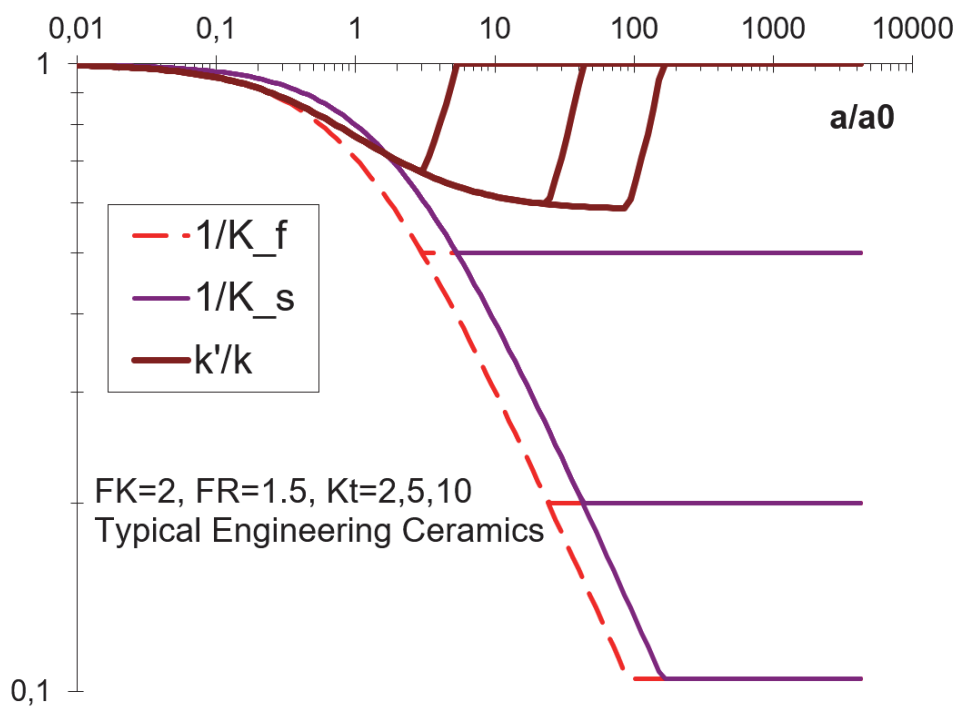

Figure 9: The generalized Wohler slope as it results from an interpolation procedure using the El Haddad equation for both the static and the fatigue criteria, and for typical material constant ratios of steels.

\section{CONCLUSIONS}

$\mathrm{O}$

ften design is a process which starts from preliminary calculations, with limited degrees of knowledge of materials and their properties. In fact this is not always only a limit of preliminary design stages, since there is never enough knowledge in fatigue of a material, except when a real prototype test is conducted, which in fact is the case for some industries, despite the larger cost of such a test with respect to analytical or numerical "virtual" testing procedures. This paper assumes that the basic Wöbler curve of the unnotched material is known, as well as the basic Paris law of the 
cracked material. We then proceed to illustrate, from the Atzori and Lazzarin criteria $[5,16]$, some simple estimates for the generic Wöbler curve of notched specimen.

\section{REFERENCES}

[1] Fleck, N.A., Kang, K.J. and Asbhy, M.F., (1994). Overview 112: The cyclic properties of engineering materials. Acta metal. mater. 42 (2), pp. 365-381.

[2] Carpinteri, A. and Karihaloo, B.L., 2003. Size-Scale Effects, Engng Fract Mech, 70(16) pp. 2255.

[3] Bazant, Z. P. (1999). Size effect on structural strength: a review, Archive of Applied Mechanics, 69, pp. 703-725.

[4] Smith, R.A. and Miller, K.J. (1978). Prediction of fatigue regimes in notched components. Int J of Mech Sci, 20, pp. 201-206.

[5] Atzori, B. and Lazzarin, P. (2001). Notch sensitivity and defect sensitivity under fatigue loading: Two sides of the same medal, Int J of Fract, 107(1), pp. L3-L8

[6] Atzori, B., Lazzarin, P. and Meneghetti, G. (2003). Fracture mechanics and notch sensitivity. Fatigue \& Fracture of Engineering Materials \& Structures, 26(3), pp. 257-267.

[7] Ciavarella, M. and Monno, F. (2006). On the possible generalizations of the Kitagawa-Takahashi diagram and of the El Haddad equation to finite life. International Journal of Fatigue, 28(12), pp. 1826-1837.

[8] Pugno, N., Ciavarella, M., Cornetti, P. and Carpinteri, A. (2006) A generalized Paris' law for fatigue crack growth. Journal of the Mechanics and Physics of Solids, 54(7), pp. 1333-1349.

[9] Ciavarella, M. (2011). Crack propagation laws corresponding to a generalized El Haddad equation. International Journal of Aerospace and Lightweight Structures (IJALS) 1, no. 1.

[10] Ciavarella, M. (2012). A simple approximate expression for finite life fatigue behaviour in the presence of "crack-like" or "blunt" notches. Fatigue \& Fracture of Engineering Materials \& Structures, 35(3), pp. 247-256.

[11] Ciavarella, M. and Papangelo, A., (2018) On the distribution and scatter of fatigue lives obtained by integration of crack growth curves: does initial crack size distribution matter? Engineering Fracture Mechanics, in press

[12] Ciavarella, M., P. D'Antuono and Papangelo, A. (2018). On the connection between Palmgren-Miner's rule and crack propagation laws. Fatigue \& Fracture of Engineering Materials \& Structures, DOI: 10.1111/ffe.12789

[13] ISO 6336:1996 Calculation of load capacity of spur and helical gears -- Part 2: Calculation of surface durability (pitting) Part 3: Calculation of tooth bending strength.

[14] Sendeckyj, G.P. (2001). Constant life diagrams — a historical review, Int J of Fatigue, 23(4), pp. 347-353.

[15] El Haddad, M.H., Topper, T.H. and Smith, K.N. (1979). Prediction of Non-Propagating Cracks. Engng Fract Mech 11, pp. 573-584.

[16] Atzori, B. and Lazzarin, P. (2000). Analisi delle problematiche connesse con la valutazione numerica della resistenza a fatica, AIAS National Conference, Lucca Italy, also Quaderno AIAS n.7, pp.33-50.

[17] Asbhy, M.F. (1989). Overview 80: The engineering properties of materials: Acta metal., 37 (5), pp. 1273-1293

[18] Asbhy, M.F. (1992). Materials selection on mechanical design, Pergamon, Oxford, UK. 